Federal Reserve Bank of Minneapolis

Research Department Working Paper 717

Revised September 2015

\title{
Equilibrium with Mutual Organizations in Adverse Selection Economies*
}

\author{
Adam Blandin \\ Arizona State University \\ John H. Boyd \\ University of Minnesota \\ Edward C. Prescott \\ Arizona State University \\ and Federal Reserve Bank of Minneapolis
}

\begin{abstract}
We develop an equilibrium concept in the Debreu (1954) theory of value tradition for a class of adverse selection economies which includes the Spence (1973) signaling and Rothschild-Stiglitz (1976) insurance environments. The equilibrium exists and is optimal. Further, all equilibria have the same individual type utility vector. The economies are large with a finite number of types that maximize expected utility on an underlying commodity space. An implication of the analysis is that the invisible hand works for this class of adverse selection economies.
\end{abstract}

Keywords: Adverse selection equilibrium; Theory of value; Insurance; Signaling; Mutual organization; The core

JEL classification: C62, D46, D82, G29, G22

*Blandin: Adam.Blandin@asu.edu; Boyd: boydx002@umn.edu; Prescott: Edward.Prescott@asu.edu. We would like to thank an anonymous referee, Rob Shimer, and Manual Amador for their useful comments. The views expressed herein are those of the authors and not necessarily those of the Federal Reserve Bank of Minneapolis or the Federal Reserve System. 


\section{Introduction}

In this paper, social insurance is defined to be the centralized provision of some form of insurance. A question is, why have social insurance? Adverse selection is often used as justification for social insurance, because many claim that adverse selection can lead to the inefficient provision of insurance by decentralized mechanisms. We find that if mutual organizations are feasible and permitted, there is no failure of decentralized arrangements in providing insurance or, for that matter, in dealing with the Akerlof (1970) used-car "lemons" environment or with the Spence (1973) job market signaling environment. If mutual insurance organizations are permitted, Adam Smith's invisible hand works. There are no market failures due to adverse selection in providing insurance.

We modify the definition of equilibrium used by Rothschild and Stiglitz (1976) in essentially one respect. We permit the agents who select insurance arrangements to select mutual arrangements. A mutual arrangement is a set of contracts which specify payments contingent on both individual experiences and the aggregate experience of all contract holders. In addition, the set of contracts must be such that the organization profits are not negative. As in Boyd-Prescott (1985), there are mutual organizations, and a core-related equilibrium concept is developed and used. But unlike this earlier approach, the new definition of equilibrium is simpler and more general. Existence, uniqueness (in the types' utility outcomes), and optimality are established for an arbitrary finite number of types of individuals. The earlier Boyd-Prescott equilibrium was defined only for two types. Attempts by us and others to generalize to more than two types have failed.

With the Rothschild-Stiglitz equilibrium definition, at the first stage nonmutual insurance contracts are selected. At the second stage, individuals choose their optimal contract from the set of offered contracts, or choose not to insure. In our definition, at the first stage mutual insurance contracts are selected. At the second stage, individuals pick their mutual arrangement optimally given the choices of others. Thus, the second stage is a Nash equilibrium.

We say that a mutual arrangement is blocked if there exists an alternative mutual arrangement for which a subset of types can make themselves better off using only their own resources. An equilibrium is defined to be an unblocked mutual arrangement. With our setup, if and only if an equilibrium mutual arrangement is chosen at stage 1 , no agent can offer an alternative mutual arrangement that is profitable, given what will happen in the second stage. ${ }^{1}$

We abstract from a number of features of reality that are important in the provision of insurance. In our examples, no costs are associated with operating an insurance company, whereas in fact operating an insurance company entails large costs. Evidence that these costs 
are large is that the gross output share of the U.S. insurance carriers and related activities sector reported in the U.S. National Accounts was 5 percent of GNP in 2012. Associated with the operation of an insurance business are record-keeping costs, monitoring costs needed to mitigate the moral hazard problems associated with insurance contracts, and asset-managing costs as premiums are received prior to claims being paid. If there were a market failure, when evaluating social insurance, these costs would have to be carefully quantified and incorporated into the analysis in addition to the costs that are specific to social insurance. But there is no need to quantify all these costs because the decentralized outcome is Pareto efficient.

\section{The Relationship to Other Equilibrium Concepts}

We use Debreu's (1954) definition of an economy. In so doing, we follow Cornet, who has been a leading contributor to the development of the theory of value (see, e.g., Cornet 1988). Debreu's definition of an economy requires the specification of a commodity space. The commodity space is a linear topological space. There also is a set of individuals with preference orderings on subsets of the commodity space and a set of technologies, which are subsets of the commodity space. Consequently, feasibility and Pareto optimality are well defined. In addition, the economic statistics of the national income accounts can be calculated and welfare analysis carried out.

An assumption is that the preference orderings of individuals can be characterized by the expected value of utility functions, which are continuous real-valued functions on the underlying consumption set. As in Prescott and Townsend (1984), the elements of the commodity space are signed measures on the Borel sigma-algebra of the underlying consumption set. The consumption sets are sets of probability measures in this space.

Equilibrium is not a Debreu valuation equilibrium as it is in the Prescott and Townsend (1984) private information economies, where households and those who pick the commodity vector in the technology sets take prices as given. Instead, as part of the definition of equilibrium, the blocking concept in Edgeworth's (1881) theory of the core is used. ${ }^{2}$ But groups do not block. Rather, an initial mutual organization is blocked if an active agent writes a charter for a new mutual organization which makes a subset of individuals better off while preserving resource and incentive feasibility. This is similar to competition among insurance firms in Rothschild and Stiglitz (1976), except that the choice is a mutual arrangement and not an insurance contract. Using our equilibrium definition, if mutual organizations are prohibited and nonmutual companies permitted, all the findings of Rothschild and Stiglitz (1976) hold. In their classic environment, for example, no transfers occur between individual types in equilibrium, and any equilibrium allocation must be the minimum separating allocation. 
Given expected utility maximization, the utility function of each individual type is linear, and the set of incentive-compatible allocations is a convex affine cone. The intersection of the resource constraint set and the incentive-compatible allocation set is a convex subset with dimension one less than the number of types.

Each person either selects the mutual organization that is best given the choices of other people or chooses not to insure. Thus, the second stage is a Nash equilibrium. The economy is large with an atomistic measure of each of the finite number of types of individuals. Type is private information.

\section{The Formal Analysis}

The economies are large with a finite number of individual types $i \in I$. The measures of type $i$ are $\lambda_{i}$. The underlying consumption sets are $C$, a closed and bounded subset of $\mathfrak{R}^{n}$. The commodity space is the space $L$ of signed measures on the Borel sigma-algebra of $C$.

Each individual's consumption possibilities set is $X \subset L$, the set of probability measures on the Borel sigma-algebra of $C$. Preferences of a type $i$ individual are ordered by the expected value of a continuous function $v_{i}$ :

$$
u_{i}(x)=\int v_{i}(c) x(d c)
$$

An allocation is an $n$-tuple $\left\{x^{i}\right\}_{i \in I}$. The incentive compatibility constraints (ICs) are

$$
u_{i}\left(x^{i}\right) \geq u_{i}\left(x^{j}\right) \text { for all } i, j \in I
$$

Type $i$ must weakly prefer $x^{i}$ to $x^{j}$.

The single resource constraint $(\mathrm{RC})$ has the form

$$
\sum_{i} \lambda_{i}\left(r_{i}\left(x^{i}\right)-\pi_{i}\right) \leq 0
$$

where the $r_{i}: X \rightarrow \mathfrak{R}$ functions are linear and the $\pi_{i}$ are parameters. In the Rothschild Stiglitz (1976) insurance case the resource constraint is the condition that a mutual insurance organization cannot distribute more in claims than it takes in as premiums. In the Spence (1973) signaling environment, it is the condition that wage payments to members of a mutual organization cannot exceed the value of production of that organization. 
An allocation is feasible if it satisfies the incentive constraints, (2), and the resource constraint, (3). An allocation $x$ is a Pareto optimum if for no other feasible allocation $y$, $u_{i}\left(y^{i}\right) \geq u_{i}\left(x^{i}\right)$ for all $i$ with strict inequality for at least one $i$.

\section{Working in the Utility Space}

We transform the problem into the utility space as the proofs of existence and optimality of unblocked mutual arrangements are carried out in this space.

Associated with each allocation $x$ is a utility vector $u(x)=\left\{u_{i}\left(x^{i}\right)\right\}_{i \in I}$. The set of utility vectors that satisfy the incentive constraints is denoted by IC. This set is a convex affine cone. The set of allocations that yield a given point in IC is convex. The mapping from utility vectors in the IC set to allocations that we use is denoted by $x(u)$. If the inverse image of $u(x)$ is not a point, then the mean allocation of all allocations that yield utility vector $u$ is the value of the function $x(u)$. This mean allocation is in the set of allocations that yield $u$, because the inverse image of the function $u(x)$ is a convex set.

The aggregate net transfer made by type $i$ is

$$
t_{i}(u)=\lambda_{i}\left(r_{i}\left(x^{i}(u)-\pi_{i}\right)\right.
$$

The resource constraint is

$$
\sum_{i} t_{i}(u)=0
$$

The set of utility vectors that satisfy this constraint is a hyper-plane with dimension one less than the cardinality of $I$.

Definition: A feasible utility vector $u$ is blocked by feasible $u^{\prime}$ if for the set of types that do better under $u^{\prime}$, denoted by $B, \sum_{i \in B} t_{i}\left(u^{\prime}\right) \geq 0$.

\section{Some Results}

Result 1: The Pareto optimum utility set, $P$, is a closed, convex subset of the hyper-plane that satisfies (5).

Result 2: Any non-Pareto optimal $u$ is blocked by the set of types $I$ and a Pareto superior feasible utility vector.

Result 3: Any unblocked $u$ is a Pareto optimum.

Result 4: Utility vector $u \in P$ is blocked if and only if there is a $u^{\prime} \in P$ that blocks it. 
Therefore, the problem is to establish the existence of an unblocked $u \in P$.

Proposition: An equilibrium exists and is unique in the utility space.

\section{Proof:}

Let $P$ be the Pareto utility set.

(i). Pick a direction in the Pareto set. Moving in that direction makes one set of types better off and the other set worse off. Thus a direction partitions the types into two sets. Moving in the given direction makes one set of types better off and the compliment set of types worse off.

(ii). A point $u$ in $P$ and a direction define a line. The intersection of a line and $P$ is a line segment.

(iii). Given a line segment in a subset in $P$, that subset can be partitioned into line segments parallel to this line segment. Along any of these partitions, utilities and transfers vary linearly. A necessary condition for an unblocked utility point to be on the line is that transfers by the benefiting group for the given direction minimizes the absolute value of the transfers by that group in that partition. The set of all utility points that satisfy this property for some partition is a convex set with dimensionality less than that of the subset. All unblocked utility points lie in that set.

(iv). Given that the dimensionality of $P$ is finite, beginning with subset $P$, by induction a set of dimension zero can be found that contains the unblocked utility points, $u^{*}$.

Comment: The allocation that yields $u^{*}$ may not be unique. If not unique, we select the allocation that is the average of the set that yields this utility outcome. Given the linearity of the mapping from feasible arrangements to utilities, the inverse image of any point in the utility possibility set is a convex set. Thus, this average is a point in the set.

\section{Application to the Adverse Selection Insurance Environment}

The environment has measures $\lambda_{i}$ of people of type $i \in\{1,2, \ldots, I\}=I$. The same symbol is used for both the cardinality of the set and the set itself. An individual's type is private information. A person has a random endowment subsequent to contracting $e_{j} \in\{1,2, \ldots, J\}=J$. The probability of a type $i$ having endowment $e_{j}$ is $\pi_{i j}$. 
The underlying consumption space is $C=\left\{x \in \mathfrak{R}_{+}^{J}: x_{j} \leq \bar{C}\right.$ for all $\left.\mathrm{j}\right\}$. The $\bar{C}$ is sufficiently large that increasing it does not increase the set of feasible allocations. This is possible because the resource constraint, necessary for feasibility, is bounded. The commodity point is

$$
x=\left\{x_{j}(d c)\right\}_{j \in J}
$$

The consumption possibility set $X$ is a vector of $J$ probability measures, and the utility functions are

$$
u_{i}(x)=\sum_{j \in J} \pi_{i j} \int v(c) x_{j}(d c) \quad i \in I
$$

The continuous function $v: C \rightarrow \mathfrak{R}$ is strictly increasing and concave.

\section{The Rothschild-Stiglitz Two-Type Adverse-Selection Environment}

There are two types and two possible positive endowments: $0<e_{b}<e_{g}$. The subscripts denote bad $(b)$ and good $(g)$. The two types are low-risk $(L)$ and high-risk $(H)$ individuals. Thus,

$$
\pi_{L b}<\pi_{H b}
$$

We normalize the population size to one so that

$$
\lambda_{H}+\lambda_{L}=1
$$

Type average endowments are

$$
\bar{e}_{i}=\pi_{i b} b+\pi_{i g} g \text { for } i \in I
$$

Depending on the parameter values, the equilibrium will fall into one of two categories: equilibria with no transfers between types, or equilibria with positive transfers from type $L$ to type $H$ individuals.

The unblocked utility vector is the one that maximizes type- $L$ utility on the utility possibility frontier. If the measure of low-risk people is sufficiently large, the set of Pareto optima is a point with everyone consuming the average population endowment $\bar{x}\left(\lambda_{L}\right)$. This average is an increasing function in $\lambda_{L}$.

For the unblocked contract, the probability measures for an $L$ type conditional on that individual endowment realization place all probability on a single point: $c_{L b}$ for $x_{b}^{L}$ and $c_{L b}$ for $x_{g}^{L}$. This follows from the assumed properties of the utility function $v$. We denote the 
consumption of a high-risk individual, which is the same for both possible endowments, by $c_{H}$. Thus, each Pareto optimum is characterized by a triplet of real numbers $\left(c_{b}, c_{g}, c_{H}\right)$. The utility functions in terms of these three variables are denoted by $U_{H}\left(c_{H}\right)=v\left(c_{H}\right)$ and $U_{L}\left(c_{b}, c_{g}\right)=\pi_{L b} v\left(c_{b}\right)+\pi_{L g} v\left(c_{g}\right)$.

To find the Pareto set, we first find the utility-maximizing contract for type $L$ given an average transfer, $T$. These transfers are constrained to the interval

$$
T \in\left[0,\left(\bar{e}_{L}-\bar{e}_{H}\right) \lambda_{H}\right]
$$

The upper end of the interval results in the average consumption of the two types being equal. The transfer for which the utility of type $L$ is maximal is the equilibrium $T^{*}$.

We proceed in two steps. First, we consider the problem of maximizing the utility of a type $L$ given $T$. The value of the solution is denoted by $\hat{u}_{L}(T)$. This program is

$$
\hat{u}_{L}(T)=\max _{c_{H}, c_{b} c_{g}} U_{L}\left(c_{b}, c_{g}\right)
$$

subject to the following constraints:

(i). The $H$ have enough resources to finance their consumption:

$$
c_{H} \leq \bar{e}_{H}+T \lambda_{L} / \lambda_{H}
$$

(ii). The $L$ have enough resources to finance their consumption:

$$
\pi_{L b} c_{b}+\pi_{L g} c_{g} \leq \bar{e}_{L}-T
$$

(iii). The $H$ weakly prefer their contract to the $L$ contract:

$$
U_{H}\left(c_{H}, c_{H}\right) \geq U_{H}\left(c_{b}, c_{g}\right)
$$

(iv). The $L$ weakly prefer their contract to the $H$ contract:

$$
U_{L}\left(c_{b}, c_{g}\right) \geq U_{L}\left(c_{H}, c_{H}\right)
$$

The curves for all fractions $\lambda_{L}$ go through the separating utility point $u^{\text {sep }}$, for which $T=0$.

To find the Pareto optimum utility sets, the utility of type $L$ is maximized given the utility level of type $H$. This function is denoted by $\hat{u}_{L}\left(u_{H}\right)$. The nature of this function depends on the fraction of low-risk types. If this fraction is sufficiently small, the curve is as depicted in 
Figure $1(a)$. The equilibrium utility vector is denoted by $u^{*}$. At this point, no transfers between types take place. The Pareto set, denoted in red, is the decreasing portion of this concave function.

(a)

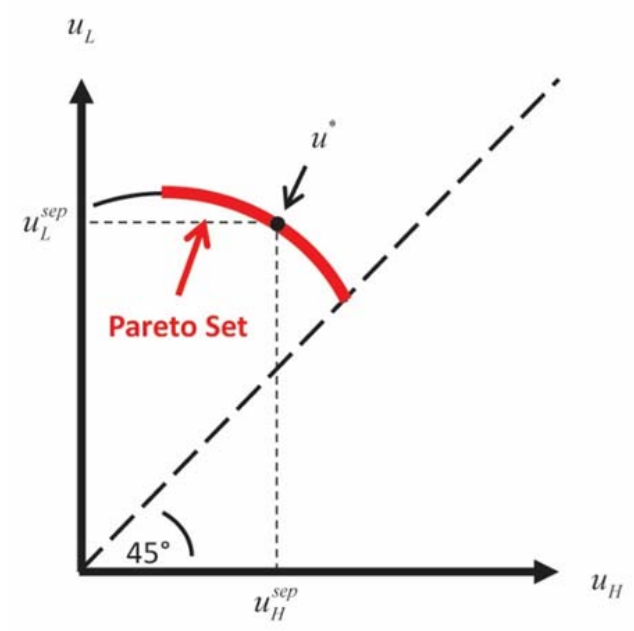

(b)

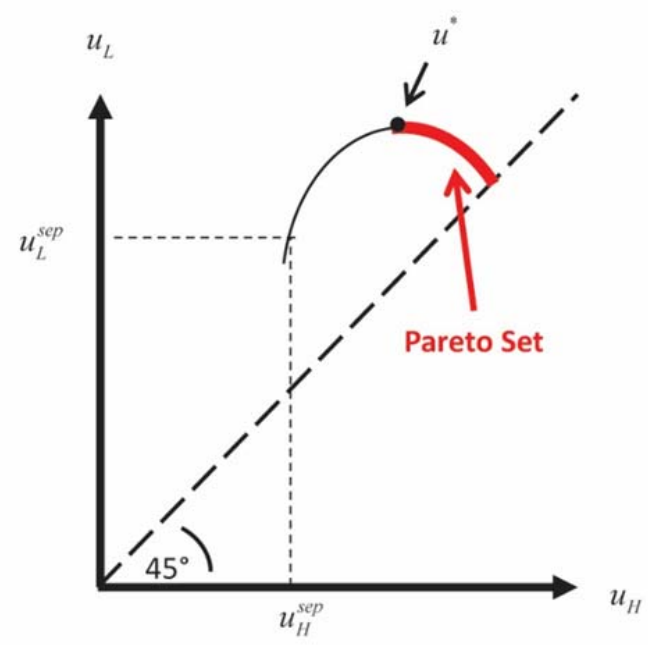

Fig. $1 \hat{u}_{L}\left(u_{H}\right)$ in the Rothschild-Stiglitz environment: a) small $\lambda_{L} ;$ b) big $\lambda_{L}$.

The greater the population fraction of the low-risk people, the higher the intersection with the 45 degree line, where all consume the mean endowment independent of type and individual endowment realization. For a sufficiently high fraction of the low-risk type, the Pareto set is a point set with everyone getting utility $v\left(\bar{e}\left(\lambda_{L}\right)\right)$ with certainty.

\section{Relation to Rothschild-Stiglitz Equilibrium}

Only for the fractions $\lambda_{L}$ for which the slope of the curve is negative at $u^{\text {sep }}$, as in Figure 1(a), does the Rothschild-Stiglitz (R-S) equilibrium exist. In these cases, the R-S equilibrium and ours are the same. But our equilibrium always exists and is unique.

\section{General Case}

The equilibrium in the more general case can be found by solving the problem of minimizing the $\ell_{1}$ norm of the transfer vector over the set of Pareto optima. The minimum exists given that it is a convex minimization problem with a compact constraint set and a 
continuous objective function. In some cases, the optimum will have nondegenerate lotteries conditional on the endowment, namely when different degrees of risk aversion can be exploited to separate the types.

\section{Application to the Spence Signaling Environment}

We turn now to the simple Spence (1973) signaling equilibrium, which does not require lotteries to convexify the economy. A point in the commodity space is $x=(c, s)$ where $c$ is consumption and $s$ is the signal and the commodity space is $\mathfrak{R}^{2}$.

Types are denoted by $i \in I=\{1,2\}$. The consumption set is $X=\left\{x \in \mathfrak{R}_{+}^{2}: x \leq x^{\max }\right\}$. Individuals of type 2 are the high-productivity individuals $\left(\pi_{2}>\pi_{1}>0\right)$ and have the lower disutility of signaling $\left(0<\theta_{2}<\theta_{1}\right)$. The utility functions are

$$
u_{i}=c-\theta_{i} s \text { for } i \in I
$$

The $x^{\max }$ is sufficiently large that all resource feasible allocations are in this consumption set. A sufficiently large $c^{\max }$ is $\pi_{2}$. A sufficient large $s^{\max }$ is $\pi_{2} / \theta_{2}$. Aggregate transfers from the high-productivity type to the low-productivity type are $t_{2}(u)=\pi_{2}-c_{2}(u)$.

The equilibrium utility vector is $u^{*}$. Figure 2(a) specifies the set of utility allocations that are incentive feasible. The set is a convex cone. Figure 2(b) specifies the feasible set of allocations and (in red) the Pareto set. For this example, the line defined by the resource constraint being binding has a negative slope, which requires the fraction of high-productivity types to be below a critical value. If it is not below this critical value, the line has a weakly positive slope, and the Pareto set is a point set with everyone consuming the population average productivity and having a zero signal.

The interesting case is the one in which the Pareto set is a downward-sloping line. Figure 2(c) specifies the sign of the transfers from the high-productivity to low-productivity people along the Pareto set. The utility vector for the equilibrium is the point $u^{*}$. 
(a)

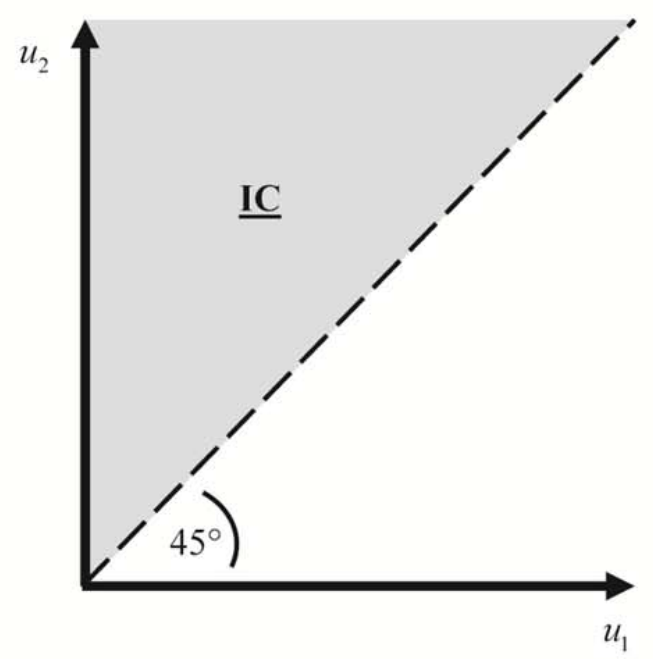

(b)

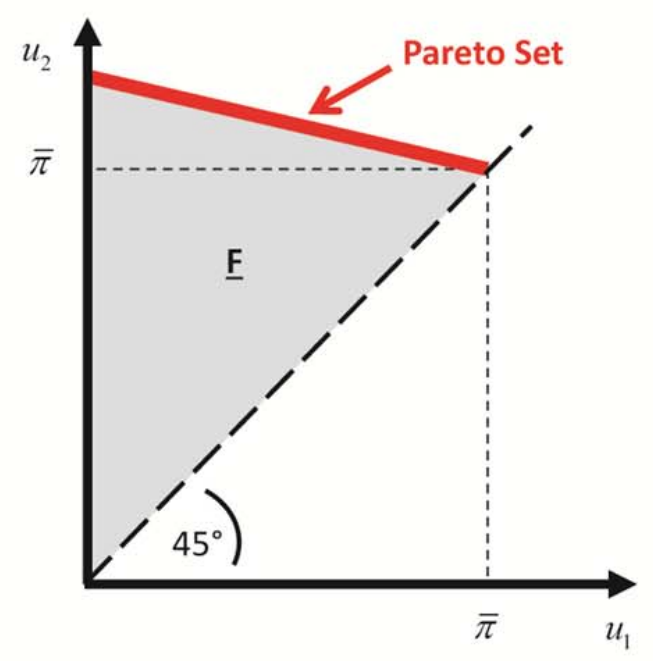

(c)

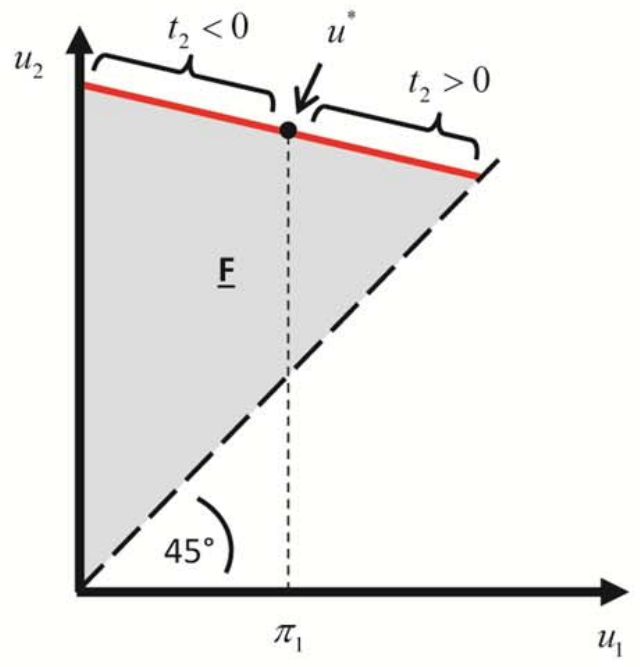

Fig. 2 The utility spaces in the Spence signaling environment: a) the IC set; b) the Pareto set; c) transfers along the Pareto set. 


\section{Relation to Spence's Valuation Equilibrium}

Spence used a valuation equilibrium with externalities. The equilibrium depends on the commodity space. The commodities were the set of signals permitted, $S$, and the consumption good. Let $c$ be the numeraire and $w_{s}$ the wages of the various types of labor. A Spence equilibrium requires that all individuals select signals that maximize their utility given the prices and, for every $s$ that was chosen by some type, the average productivity of those who choose that $s$ is equal to $w_{s}$.

Any feasible allocation is an equilibrium for an appropriately selected $S$, as was shown in Prescott and Townsend (1981). For each signal, the associated wage is the average productivity of people who choose that signal.

\section{Concluding Comments}

The equilibrium concept developed requires there be a single resource constraint and that individuals maximize expected utility. This is an important class of environments that have received a great deal of attention in the economics literature. Equilibrium concepts that are useful in some environments are not useful in others. We see this equilibrium as expanding the set of environments for which there is a useful equilibrium concept. 


\section{References}

Akerloff, G. A. (1970). The market for "lemons": Quality uncertainty and the market mechanism. Quarterly Journal of Economics, 84(3), 488-500.

Boyd J. H., and E. C. Prescott (1985). Financial intermediary-coalitions. Staff Report 87, Federal Reserve Bank of Minneapolis.

Cornet, B. (1988). General equilibrium theory and increasing returns: Presentation. Journal of Mathematical Economics, 17(2-3), 103-118.

Debreu, G. (1987). Theory of value: An axiomatic analysis of economic equilibrium. Monograph 17. New Haven, CT: Yale University Press.

Debreu, G. (1954). Valuation equilibrium and Pareto optimum. Proceedings of the National Academy of Sciences of the United States of America, 40(7), 588-592.

Debreu, G., and H. Scarf (1963). A limit theorem on the core of an economy. International Economic Review, 4(3), 235-246.

Dubey, P., and J. Geanakoplos (2002). Competitive pooling: Rothschild-Stiglitz reconsidered. Quarterly Journal of Economics, 117(4), 1529-1570.

Edgeworth, F. Y. (1881). Mathematical psychics: An essay on the application of mathematics to the moral sciences. London: C. Kegan Paul.

Foley, D. K. (1974). Lindahl's Solution and the core of an economy with public goods. Econometrica, 38(1), 66-72.

Mas-Colell, A. (1982). Perfect competition and the core. Review of Economic Studies, 49(1), 1530.

Ostroy, J. M. (1980). The no-surplus condition as a characterization of perfectly competitive equilibrium. Journal of Economic Theory, 22(2), 183-207.

Prescott, E. C., and R. M. Townsend (1981). Optima and competitive equilibria with adverse selection and moral hazard. Discussion Paper No. 81-152, University of Minnesota.

Prescott, E. C., and R. M. Townsend (1984). Pareto optima and competitive equilibria with adverse selection and moral hazard. Econometrica 52(1): 21-45. 
Rothschild, M., and J. Stiglitz (1976). Equilibrium in competitive insurance markets: An essay on the economics of imperfect information. Quarterly Journal of Economics, 90(4), 629-649.

Smith, A. (1937). The wealth of nations. Modern Library Edition. New York: Random House. (Orig. pub. 1776.)

Spence, M. (1973). Job market signaling. Quarterly Journal of Economics, 87(3), 355-374.

Wilson, C. A. (1979). Equilibrium and adverse selection. American Economic Review, 69(2), 313317.

Wilson, R. (1968). The theory of syndicates. Econometrica, 36(1), 119-132.

\footnotetext{
${ }^{1}$ The no-profit condition is related to Ostroy's (1980) no-surplus condition.

${ }^{2}$ Debreu and Scarf (1963) show that with convexity, under replication, in the limit the set of core allocations is the set of valuation equilibria.
} 